\title{
GENERATING FUNCTIONS OF APPELL FORM FOR THE CLASSICAL ORTHOGONAL POLYNOMIALS ${ }^{1}$
}

\author{
R. C. T. SMITH
}

1. Summary. A sequence of polynomials $P_{n}(x)$ (of degree $n$ ) will be said to be of Appell type if it can be represented by a generating function of the form

$$
K(x, w)=\sum_{n=0}^{\infty} w^{n} P_{n}(x)=A(g(w)) \psi(x g(w))
$$

with

$$
\begin{aligned}
\text { (i) } g(w) & =g_{1} w+g_{2} w^{2}+\cdots, \\
\text { (ii) } A(y) & =1+a_{1} y+a_{2} y^{2}+\cdots, \\
\text { (iii) } \psi(t) & =1+\psi_{1} t+\psi_{2} t^{2}+\cdots,
\end{aligned}
$$

where the coefficients are constants.

We have determined all generating functions of Appell form for the classical orthogonal polynomials (Hermite, Jacobi, Laguerre). ${ }^{2}$

It appears that all these results are known and consequently the formulae will not be reproduced here.

For Jacobi polynomials our results may be stated in the form

Theorem. The Jacobi polynomials $P_{n}^{(\alpha, \beta)}(x)$ are of Appell type only if the difference $\alpha-\beta$ takes one of the values $-1,0,1$.

2. Method. The polynomials we consider all satisfy differential equations of the form

$$
L\left(x, x \frac{d}{d x}, n\right) y=0
$$

where

$$
\begin{aligned}
L= & \left(\alpha_{0}+\frac{\alpha_{1}}{x}+\frac{\alpha_{2}}{x^{2}}\right)\left(x \frac{d}{d x}\right)^{2}+\left(\beta_{0}+\frac{\beta_{1}}{x}-\frac{\alpha_{2}}{x^{2}}\right)\left(x \frac{d}{d x}\right) \\
& +\gamma_{0} n^{2}+\delta_{0} n .
\end{aligned}
$$

Received by the editors April 4, 1955 and, in revised form, May 24, 1955 and July 7, 1955 .

${ }^{1}$ Professor R. C. Buck raised the question, whether Jacobi polynomials are of Appell type or not, in connection with his work on expansion theorems for analytic functions. The author wishes to thank Professor Buck for several helpful discussions.

2 We follow the notation used by Szegö [1]. 
Consequently the generating function

$$
K(x, w)=\sum_{n=0}^{\infty} w^{n} P_{n}(x)
$$

satisfies

$$
L\left(x, x \frac{\partial}{\partial x}, w \frac{\partial}{\partial w}\right) K=0 .
$$

On changing the variables to

$$
u=w, \quad t=x g(w)
$$

and using the relations

$$
\begin{aligned}
& \left(\frac{\partial}{\partial w}\right)_{x}=\left(\frac{\partial}{\partial u}\right)_{t}+x g^{\prime}(w)\left(\frac{\partial}{\partial t}\right)_{u}, \\
& \left(\frac{\partial}{\partial x}\right)_{w}=g(w)\left(\frac{\partial}{\partial t}\right)_{u}
\end{aligned}
$$

we obtain

$$
L\left(\frac{t}{g(u)}, t \frac{\partial}{\partial t}, u \frac{\partial}{\partial u}+\left(\frac{u g^{\prime}(u)}{g(u)}\right) t \frac{\partial}{\partial t}\right) K=0 .
$$

This can be rewritten in the form

$$
L\left(\frac{t}{g(u)}, t \frac{\partial}{\partial t}, \frac{u g^{\prime}(u)}{g(u)}\left\{g(u) \frac{\partial}{\partial g(u)}+t \frac{\partial}{\partial t}\right\}\right) K=0 .
$$

From (2) (i)

$$
G(u)=\frac{u g^{\prime}(u)}{g(u)}=1+G_{1} g+G_{2} g^{2}+\cdots
$$

where $G_{1}, G_{2}, \cdots$ are constants. Any relation of the form (8) (satisfying suitable convergence conditions) can be solved to give $g(u)$ in the form (2) (i).

Substituting (4) in ( 7$)$ and writing $\theta=g(\partial / \partial g)$ we obtain

$$
\begin{aligned}
& {\left[\left(\alpha_{0}+\frac{\alpha_{1} g}{t}+\frac{\alpha_{2} g^{2}}{t^{2}}\right)\left(t \frac{\partial}{\partial t}\right)^{2}+\left(\beta_{0}+\frac{\beta_{1} g}{t}-\frac{\alpha_{2} g^{2}}{t^{2}}\right)\left(t \frac{\partial}{\partial t}\right)\right.} \\
& \left.\quad+\gamma_{0} G\left(\theta+t \frac{\partial}{\partial t}\right) G\left(\theta+t \frac{\partial}{\partial t}\right)+\delta_{0} G\left(\theta+t \frac{\partial}{\partial t}\right)\right] K=0 .
\end{aligned}
$$

From (1) and (2) (iii) 


$$
K=A(g) \sum_{n=0}^{\infty} \psi_{n} t^{n}
$$

Substituting in (9) and equating the coefficient of $t^{n}$ to zero we obtain the recurrence relations

$$
\begin{aligned}
{\left[n ^ { 2 } \left(\alpha_{0}\right.\right.} & \left.+\gamma_{0} G^{2}\right) A+n\left\{\beta_{0} A+\gamma_{0}\left(2 G^{2} \theta A+G A \theta G\right)+\delta_{0} G A\right\} \\
& \left.+\gamma_{0}\left(G^{2} \theta^{2} A+G \theta G \theta A\right)+\delta_{0} G \theta A\right] \psi_{n} \\
& +\left[(n+1)\left\{\alpha_{1}(n+1)+\beta_{1}\right\} A g\right] \psi_{n+1} \\
& +\left[(n+2)(n+1) \alpha_{2} A g^{2}\right] \psi_{n+2}=0 \quad \text { for } n=0,1,2, \cdots
\end{aligned}
$$

Solving from the first three of these equations shows that

$$
\alpha_{0} A+\gamma_{0} A G^{2}=\lambda_{1} A g+\lambda_{2} A g^{2},
$$

(ii) $\beta_{0} A+\gamma_{0}\left(2 G^{2} \theta A+G A \theta G\right)+\delta_{0} G A=\mu_{1} A g+\mu_{2} A g^{2}$,

(iii) $\gamma_{0}\left\{G^{2} \theta^{2} A+G \theta G \theta A\right\}+\delta_{0} G \theta A=\nu_{1} A g+\nu_{2} A g^{2}$,

where $\lambda_{1}, \lambda_{2}, \mu_{1}, \mu_{2}, \nu_{1}, \nu_{2}$ are constants depending on $\psi_{1}, \psi_{2}, \psi_{3}, \psi_{4}$. Substituting in (10)

$$
\begin{aligned}
& A g\left[\left(n^{2} \lambda_{1}+n \mu_{1}+\nu_{1}\right) \psi_{n}+(n+1)\left\{(n+1) \alpha_{1}+\beta_{1}\right\} \psi_{n+1}\right] \\
& +A g^{2}\left[\left(n^{2} \lambda_{2}+n \mu_{2}+\nu_{2}\right) \psi_{n}+(n+2)(n+1) \alpha_{2} \psi_{n+2}\right]=0 .
\end{aligned}
$$

This gives two independent recurrence relations

(i) $(n+1)\left\{(n+1) \alpha_{1}+\beta_{1}\right\} \psi_{n+1}=-\left\{n^{2} \lambda_{1}+n \mu_{1}+\nu_{1}\right\} \psi_{n}$,

$$
(n+2)(n+1) \alpha_{2} \psi_{n+2}=-\left\{n^{2} \lambda_{2}+n \mu_{2}+\nu_{2}\right\} \psi_{n}
$$

except in exceptional cases. These recurrence relations will be inconsistent unless the constants $\alpha_{1}, \alpha_{2}, \beta_{1}, \lambda_{1}, \lambda_{2}, \mu_{1}, \mu_{2}, \nu_{1}, \nu_{2}$ satisfy certain conditions.

From (11) we easily obtain two necessary conditions for the polynomials $P_{n}(x)$ satisfying (4) to be Appell polynomials. Since $A=1$, $G=1$ when $g=0$,

$$
\alpha_{0}+\gamma_{0}=0, \quad \beta_{0}+\delta_{0}=0 .
$$

These conditions are satisfied by the Jacobi, Hermite and Laguerre polynomials.

From here on we assume the conditions (13) to be satisfied so that the relations (11) take the form

$$
\alpha_{0}\left(1-G^{2}\right)=\lambda_{1} g+\lambda_{2} g^{2},
$$

(ii) $\alpha_{0}\left(2 G^{2} \theta A+G A \theta G\right)-\beta_{0}(1-G) A=-\left(\mu_{1} g+\mu_{2} g^{2}\right) A$,

(iii) $\quad \alpha_{0}\left(G^{2} \theta^{2} A+G \theta G \theta A\right)+\beta_{0} G \theta A=-\left(\nu_{1} g+\nu_{2} g^{2}\right) A$. 
In general these three equations for the two unknowns $A(g), g(u)$ will not be consistent. Our problem is to determine $\lambda_{1}, \lambda_{2}, \mu_{1}, \mu_{2}, \nu_{1}, \nu_{2}$ so that (12)(i), (ii), (14)(i), (ii), (iii) are consistent, if this is possible, and then to solve for $A(g), g(u), \psi(t)$. For certain values of $\alpha_{0}, \alpha_{1}, \alpha_{2}$, $\beta_{0}, \beta_{1}$ one of the recurrence relations (12)(i), (ii) or one of the differential equations (14)(i), (ii), (iii) may disappear.

3. Jacobi polynomials $P_{n}^{(\alpha, \beta)}(x)$. The associated differential equation is

$$
\begin{aligned}
& \left\{\left(1-\frac{1}{x^{2}}\right)\left(x \frac{d}{d x}\right)^{2}\right. \\
& \left.\quad+\left(\alpha+\beta+1+\frac{\alpha-\beta}{x}+\frac{1}{x^{2}}\right)\left(x \frac{d}{d x}\right)-n(n+\alpha+\beta+1)\right\} y=0
\end{aligned}
$$

and so we must replace $\alpha_{0}$ by $1, \alpha_{1}$ by $-1, \alpha_{2}$ by $-1, \beta_{0}$ by $\alpha+\beta+1$ and $\beta_{1}$ by $\alpha-\beta$. We assume $\alpha-\beta \neq 0$.

From (12)(i)

$$
\begin{aligned}
(n+2)(n+1) & (\alpha-\beta)^{2} \psi_{n+2} \\
= & \left\{(n+1)^{2} \lambda_{1}+(n+1) \mu_{1}+\nu_{1}\right\}\left\{n^{2} \lambda_{1}+n \mu_{1}+\nu_{1}\right\} \psi_{n}
\end{aligned}
$$

which is only consistent with (12)(ii) if

$$
\begin{aligned}
\left\{(n+1)^{2} \lambda_{1}+(n+1) \mu_{1}+\nu_{1}\right\} & \left\{n^{2} \lambda_{1}+n \mu_{1}+\nu_{1}\right\} \\
& =\left\{n^{2} \lambda_{2}+n \mu_{2}+\nu_{2}\right\}(\alpha-\beta)^{2} .
\end{aligned}
$$

Obviously $\lambda_{1}=0$ and (15) further simplifies to

$$
\left\{(n+1) \mu_{1}+\nu_{1}\right\}\left\{n \mu_{1}+\nu_{1}\right\}=\left\{n^{2} \lambda_{2}+n \mu_{2}+\nu_{2}\right\}(\alpha-\beta)^{2} .
$$

Comparing coefficients of $n^{0}, n, n^{2}$

(16) ${ }_{\mu_{1}}^{2}=(\alpha-\beta)^{2} \lambda_{2}, \mu_{1}^{2}+2 \mu_{1} \nu_{1}=(\alpha-\beta)^{2} \mu_{2}, \mu_{1} \nu_{1}+\nu_{1}^{2}=(\alpha-\beta)^{2} \nu_{2}$.

From (14)(ii), $2 \theta A G^{2}=A\left\{\beta_{0}(1-G)-G \theta G-\mu_{1} g-\mu_{2} g^{2}\right\}$. Operating on both sides with $\theta$ we obtain

$$
\begin{aligned}
2 \theta^{2} A G^{2}+4 \theta A G \theta G & =-\theta A\left\{G \theta G-\beta_{0}(1-G)+\mu_{1} g+\mu_{2} g^{2}\right\} \\
& +A\left\{-\beta_{0} \theta G-(\theta G)^{2}-G \theta^{2} G-\mu_{1} g-2 \mu_{2} g^{2}\right\} .
\end{aligned}
$$

Therefore

$2 \theta^{2} A G^{2}+2 \theta A G \theta G+2 \theta A \beta_{0} G$

$$
\begin{aligned}
= & -\theta A\left\{3 G \theta G-\beta_{0}(1+G)+\mu_{1} g+\mu_{2} g^{2}\right\} \\
& +A\left\{-\beta_{0} \theta G-(\theta G)^{2}-G \theta^{2} G-\mu_{1} g-2 \mu_{2} g^{2}\right\} .
\end{aligned}
$$


For this to be consistent with (14)(iii) we must have

$$
\begin{aligned}
\theta A\{3 G \theta G & \left.-\beta_{0}(1+G)+\mu_{1} g+\mu_{2} g^{2}\right\} \\
& =A\left\{-\beta_{0} \theta G-(\theta G)^{2}-G \theta^{2} G+\left(2 \nu_{1}-\mu_{1}\right) g+\left(2 \nu_{2}-2 \mu_{2}\right) g^{2}\right\}
\end{aligned}
$$

which reduces to

$$
\begin{aligned}
& \text { (17) }\left\{-G \theta G+\beta_{0}(1-G)-\mu_{1} g-\mu_{2} g^{2}\right\}\left\{3 G \theta G-\beta_{0}(1+G)+\mu_{1} g+\mu_{2} g^{2}\right\} \\
& =2 G^{2}\left\{-\beta_{0} \theta G-(\theta G)^{2}-G \theta^{2} G+\left(2 \nu_{1}-\mu_{1}\right) g+\left(2 \nu_{2}-2 \mu_{2}\right) g^{2}\right\}
\end{aligned}
$$

on again using the expression for $\theta A$ and removing the factor $A$.

On substituting $\lambda_{1}=0$, (14)(i) becomes

$$
G^{2}=1-\lambda_{2} g^{2}
$$

which gives

$$
\begin{aligned}
G \theta G & =-\lambda_{2} g^{2}, \\
G \theta^{2} G+(\theta G)^{2} & =-2 \lambda_{2} g^{2} .
\end{aligned}
$$

Substituting these relations in (17) gives

$$
\begin{aligned}
g\left\{2 \mu_{1}\left(\beta_{0}+1\right)\right. & \left.-4 \nu_{1}\right\} \\
& +g^{2}\left\{-\lambda_{2}\left(\beta_{0}+2\right)^{2}+2 \mu_{2}\left(\beta_{0}+2\right)-4 \nu_{2}-\mu_{1}^{2}\right\} \\
& +g^{3}\left\{2 \mu_{1} \lambda_{2}-2 \mu_{1} \mu_{2}+4 \nu_{1} \lambda_{2}\right\} \\
& +g^{4}\left\{\lambda_{2}^{2}-\mu_{2}^{2}+4 \lambda_{2} \nu_{2}\right\}=0 .
\end{aligned}
$$

Now substituting the relations (16) in (18) we obtain finally

$$
\begin{aligned}
& g\left\{2(\alpha+\beta+2) \mu_{1}-4 \nu_{1}\right\} \\
& \quad+\left(g^{2} /(\alpha-\beta)^{2}\right)\left\{-\mu_{1}^{2}(\alpha+\beta+3)^{2}+2\left(\mu_{1}^{2}+2 \mu_{1} \nu_{1}\right)(\alpha+\beta+3)\right. \\
& \left.\quad-4\left(\mu_{1} \nu_{1}+\nu_{1}^{2}\right)-\mu_{1}^{2}(\alpha-\beta)^{2}\right\}=0 .
\end{aligned}
$$

Equating the coefficient of $g$ to zero we find

$$
2 \nu_{1}=(\alpha+\beta+2) \mu_{1} \text {. }
$$

Substituting this value of $\nu_{1}$ we find, as the coefficient of $g^{2}$

$$
\frac{\stackrel{2}{\mu_{1}}}{(\alpha-\beta)^{2}}\left\{1-(\alpha-\beta)^{2}\right\}
$$

which vanishes for

$$
\alpha-\beta= \pm 1
$$


Without significant loss of generality we may take $\mu_{1}=1$. Determining $g(u), A(y), \psi(t)$ from the differential equations (14)(i), (ii) and the recurrence relation (12)(i) leads to known generating functions.

For $\alpha-\beta=0$ we have the ultraspherical polynomials with known generating functions of Appell type.

\section{REFERENCE}

1. G. Szegö, Orthogonal polynomials, Amer. Math. Soc. Colloquium Publications, vol. $23,1939$.

\section{APPROXIMATION BY FAMILIES OF FUNCTIONS ${ }^{1}$}

\section{LEONARD TORNHEIM}

Let $F$ be a set of real functions on the interval $a \leqq x \leqq b$ and let $g(x)$ be a real and continuous function on the same interval. The problem of finding a function in $F$ which approximates $g$ best according to some criterion has been extensively studied, especially in the two cases when $F$ is the set of all real polynomials of degree less than $n$ or a fixed set of trigonometric functions $[1 ; 2 ; 4$, pp. 40-41]. We investigate the problem for $n$-parameter families of functions [5] with reference to some of the usual approximation criteria.

An $n$-parameter family $F$ of functions on the interval $a \leqq x \leqq b$ is a set of real continuous functions $f$ such that for every set of points $\left(x_{i}, y_{i}\right)(i=1, \cdots, n)$ with $a \leqq x_{1}<\cdots<x_{n} \leqq b$ there exists exactly one $f$ in $F$ with $f\left(x_{i}\right)=y_{i}(i=1, \cdots, n)$.

An $n$-parameter family is linear if it is a real vector space, i.e., if there exist $n$ functions $f_{1}, \cdots, f_{n}$ such that every $f$ may be expressed as a linear combination $f=a_{1} f_{1}+\cdots+a_{n} f_{n}$, where $a_{1}, \cdots, a_{n}$ are real.

For $k \geqq 1$ the modulus of approximation $M^{(k)}$ of $g$ by $f$ is defined as

$$
\begin{array}{ll}
M^{(k)}(f) & =\int_{a}^{b}|f-g|^{k} d x \quad(k<\infty), \\
M^{(\infty)}(f) & =\max |f-g| .
\end{array}
$$

Further a function $f_{0}$ in $F$ will be called a best $k$-approximant to $g$ if

Presented to the Society, November 27, 1954; received by the editors August 1, 1955.

${ }^{1}$ Supported by a grant from the National Science Foundation. 